\title{
Nano formulated proanthocyanidins as an effective wound healing component
}

\author{
R. Rajakumari a, b, Tatiana Volovab, Oluwatobi Samuel Oluwafemi*c, d, Rajesh Kumare, Sabu
}

Thomasa, f and Nandakumar Kalarikkal*a, g

R. Rajakumari a, b, Tatiana Volovab, Oluwatobi Samuel Oluwafemi* ${ }_{c}$, d, Rajesh Kumare, Sabu Thomasa, f and Nandakumar Kalarikkal*a, g

aInternational and Inter-University Centre for Nanoscience and Nanotechnology, Mahatma

Gandhi University, Kerala, 686560, India

bSiberian Federal University, 79 Svobodnyi Av., Krasnoyarsk, 660041, Russia

E-mail:volova45@mail.ru

c Department of Applied Chemistry, University of Johannesburg, South Africa

dCentre for Nanomaterials Sciences Research, University of Johannesburg, Johannesburg,

South Africa

E-mail: oluwafemi.oluwatobi@gmail.com

eDepartment of Pharmacology, Saveetha Dental College and Hospitals, Saveetha Institute of

Medical and Technical Sciences (SIMATS), Chennai, 600077, Tamilnadu, India

E-mail: ssrajeshkumar@hotmail.com,Tel: +9196297 39263

fSchool of Chemical Sciences, Mahatma Gandhi University, Kerala, 686560, India.

E-mail: sabuchathukulam@yahoo.co.uk, Fax: +91-481-2731002, Tel: +91-481-2731002

${ }_{g}$ School of Pure and Applied Physics, Mahatma Gandhi University, Kerala, 686560, India.

E-mail: nkkalarikkal@mgu.ac.in,Fax: +91-481-2731669, Tel: +91-481-2731669

\begin{abstract}
Proanthocyanidins (PCs), a component of grape seed extract (GSE), has recently being used for the treatment of wounds. However, poor absorption, poor stability and rapid elimination from the systemic circulation limit its acceptance. In addressing these problems, we herein report the development of PCs based nanoformulations (PCs/SOLU) for the first time based on $1 \%$ GSE and assessed its wound healing potential in-vivo on the wistar rats. GSE and PCs/SOLU nanodispersions $1 \%$ were prepared by incorporating them into the ointment base via uniform mixing to form ointment which could be easily applied topically to wounds. The antibacterial activity of PCs/SOLU against gram positive and gram-negative bacteria strains proved that the cell membranes became more permeable with disrupted cell structure. While carrageenan and histamine induced rat paw edema analyses show there was no inflammatory signs in animals treated with $1 \mathrm{wt} \%$ of PCs/SOLU nanodispersion. Excision wound measuring about $3 \mathrm{~cm}$ in depth was created on the wistar rats. The ointment was applied topically on the wounded site and the wound contraction was measured daily. Grape seed extract (GSE) ointment, ointment base and povidone-iodine (Povi-Iod) ointment of about $1 \%$ was used as the control, positive and negative standards. PCs/SOLU nanodispersion heals the wound by mobilising the fibroblasts in the wound site and inhibits the inflammatory response through decreased expression of monocyte. The macroscopical, immunological and histopathological assessments revealed that PCs/SOLU nanodispersion ointment usage improves the cell adhesion and proliferation
\end{abstract}

\section{Introduction}

Wound healing is formed by series of events such as proliferation and migration of cells in which the migration of cells occurs from blood components such as thrombocytes, macrophages and neutrophils. There are three important process required for successful wound healing such as fibroplasia, angiogenesis and re-epithelialisation. Fibroplasia is the stage in 
which the wound contraction occurs by the process of proliferation of fibroblasts[1,2].

Angiogenesis and re-epithelisation are the stages in which the wound contraction occurs in storing the matrix for the formation of new vein and epithelium. A delay in the development of these mechanisms leads to failure or prolong wound healing process[3- 8].

There are various topical and systemic agents used for the cure of wound and antimicrobial agents which are effective in preventing infections in surgical and traumatic wounds[9- 12 . However, these topical agents delays re-epithelialisation and might pose a risk of oversensitivity and resistance[13-15]. For this reason, some alternative wound treatment methods have been investigated. Among these, the applications of plant based nanotechnology plays a significant role in the process of wound healing[16,17]. The plant based materials have proved at all times to be a powerful remedy for health care[18- 27]. Accordingly, the grape seed extract containing PCs have been investigated for wound treatment and in the management of many illnesses such as cancers, cardiovascular disease, gastric ulcers, obesity and skin inflammations. PCs also helps in promoting the formation of collagen and elastin and preservation of skin moisture. Furthermore, PCs induces vascular endothelial growth factor, reduces edema and encourages blood flow thereby accelerates wound healing[28- 33]. Khanna et al reported the outcome of grape seed extract (GSE) on in-vivo wound healing among nine male BalbC mice. They revealed that extract containing $2 \%$ of resveratrol accelerated wound healing $(\mathrm{P}<0.05)$. They also found that improved histological structure, deposition of connective tissue and increased proliferation stimulated the production of VEGF (vascular endothelial growth factor) at the wound edge[34]. In a work by Hemmati et al, they found that $2 \%$ GSE enhanced the process of wound contraction and closure in rabbits, and reported a wound healing within 15 days[32]. In an another study, they have used a grape skin powder on full thickness skin lesions in mice and observed a wound healing occurred thirteen days after treatment begun [35]. The findings of their clinical study indicate that they have used $5 \%$ ointment of GSE on caesarean section (CS). They observed that it accelerates wound healing and reduce its closure time[31]. The findings of all these reported studies confirms that they have used about 2- $5 \%$ of GSE without using any polymer. These works also reported poor stability and rapid elimination of GSE from systemic circulation.

In addressing these problems, we have developed the PCs based nanoformulations for the first time based on $1 \%$ GSE and assessed its wound healing potential. The outcomes of the current study indicate that $1 \%$ of PCs/SOLU nanodispersion ointment of grape seed extract can accelerate excision wound healing and helps to eliminate the complications associated with the wounds such as inflammation and infections. In addition, the PCs/SOLU nanodispersion ointment can acts as an antioxidant, antibacterial and anti-inflammatory agent in-vivo and thus improved regeneration process in wounds.

\section{Materials}

Grape seed extract (GSE) used in this study was purchased from JF naturals, China.

Polyethylene glycol-polyvinyl acetate-polyvinyl caprolactam (Soluplus (SOLU)) were received from BASF Corporation, Mumbai. Hard paraffin, yellow soft paraffin, wool fat and cetosteryl alcohol were obtained from Sigma Aldrich, Mumbai. Hematoxylin, eosin, acetone, glacial acetic acid, xylene, $\mathrm{NaCl}, \mathrm{KCl}, \mathrm{Na}_{2} \mathrm{HPO}_{4}$ and $\mathrm{K}_{2} \mathrm{HPO}_{4}$ were procured from Merck, India. Formaldehyde was purchased from Fisher Scientific, India. The bacterial strains such as E.coli (ATCC25922) and S.aureus (ATCC 25923) were obtained from Saveetha Medical College, India. All the chemicals used were of analytical grade.

\section{Methods}

\section{Freeze-drying}

The different ratios of PCs/SOLU (1:1 (FI), 1:3 (FII), 1:5 (FIII), 1:7 (FIV)) were dissolved in distilled water and the prepared solution was dispersed using magnetic stirrer at $125 \mathrm{rpm}$ for 
$30 \mathrm{~min}$. Then, it was sonicated for $10 \mathrm{mins}$ at $25^{\circ} \mathrm{C}$ and the solution was frozen at $-45^{\circ} \mathrm{CFinally,}$ the frozen sample was subjected to lyophilisation at $-84^{\circ} \mathrm{C}$ with a pressure of $7 \times 10-2$ mbar for $12 \mathrm{~h}$ using a freeze-dryer (Sub-Zero, USA) to obtain the dried solid.

\section{Loading Efficiency}

The percentage of PCs dispersed in SOLU was determined by membrane filtration method. $\mathrm{PCs} / \mathrm{SOLU}$ of about $1 \mathrm{ml}$ was filtered through the membrane with pore size of $0.22-\mu \mathrm{m}$, while non-dispersed PCs was retained in the membrane.

\section{Characterization}

The functional groups present in GSE, SOLU and GSE-SOLU dispersions were measured by Perkin Elmer FT-IR spectrometer in the frequency of 4000-500 cm-1 in attenuated total reflectance mode. The interactions between the PCs and SOLU were also investigated by FTIR measurements. The obtained spectra was baseline corrected by using Perkin Elmer Spectrum software. D8-Advance of Bruker XRD (Germany) was used to determine the crystallinity and the structure of the GSE, SOLU and PCs-SOLU dispersions with wavelength of $1.5405980 \AA$. The morphological analysis was carried out using JEOL Model-JEM2100 transmission electron microscope (TEM). GSE, SOLU and GSE-SOLU dispersions were dissolved in water and analysed using JascoV-650 absorption spectrometer. Horiba nano zetasizer (UK) was used to measure the particle size distribution, polydispersity index (PDI) and zeta potential of GSE, SOLU and GSE-SOLU dispersions.

\section{In-vitro release assay}

The disso profile of PCs from SOLU was estimated using SIF (Simulated Intestinal Fluid). For the preparation of SIF, Wang et al's procedure was adopted by mixing trypsin - $10 \mathrm{~g} / \mathrm{l}, \mathrm{NaCl}-9 \mathrm{~g} / \mathrm{l}$, pancreatin - $10 \mathrm{~g} / \mathrm{l}$ and bile salts $-3 \mathrm{~g} / \mathrm{l}$. The $\mathrm{pH}$ of the fluid was adjusted to 6.8 [36]. Then the prepared fluid was incubated at $37^{\circ} \mathrm{C}$ and continuously stirred using magnetic stirrer.

During the initial $6 \mathrm{~h}$ period, $1 \mathrm{ml}$ of the solution was withdrawn at $1 \mathrm{~h}$ interval and after $6 \mathrm{~h}$ aliquots were taken at every $3 \mathrm{~h}$ until $12 \mathrm{~h}$ period. After which aliquots were also taken at every $8 \mathrm{~h}$ until $48 \mathrm{~h}$. The withdrawn solutions was centrifuged at 14,000 rpm for 5 min and filtered using $0.45 \mu \mathrm{m}$ Millipore. The PCs content was estimated by HPLC (Waters HPLC). GSE (10 $\mathrm{ml})$ was mixed in acetone/water/acetic acid (70/29.5/0.5 v/v/v) and this solution was filtered through $0.45 \mu \mathrm{m}$ PVDF membrane. $10 \mu \mathrm{l}$ of the solution was injected and the stationary phase used here was $5 \mu \mathrm{m}, 250 \times 4.6 \mathrm{~mm}$ silica column. Finally, the components were detected by fluorescence spectrometer. The obtained standard and the sample peaks were quantified and expressed in PCs $\mathrm{mg} / \mathrm{g}$ of GSE.

\section{Antibacterial studies}

The qualitative and quantitative assay of the prepared nano dispersions was carried out using agar well diffusion method. The in-vitro antibacterial assay was performed based on the standards protocol given by National Committee for Clinical Laboratory Standards (NCCLS) protocol. The gram positive and gram-negative strains were used (E.coli, Bacillus and $S$. aureus). The samples analysed included the optimised PCs/SOLU nanodispersion (FIII), GSE and standard. The bacterial suspension (inoculum) was prepared by using Mac Farland (108 $\mathrm{CFU} / \mathrm{ml}$ ) media. In the Mueller Hinton agar medium, the prepared inoculum was streaked over the surface and dried. The wells were created using sterile borer and the samples (Gentamycin, GSE, PCs-SOLU) dissolved in water was poured into the wells. Then the plates were kept for incubation at $37^{\circ} \mathrm{C}$ for $24 \mathrm{~h}$. The activity was measured by zone of inhibition developed around the wells. Finally, the images of all the plates were taken after developing zone of inhibitfluorescence spectrometer. The obtained standard and the sample peaks were quantified and expressed in PCs mg/g of GSE.

\section{Antibacterial studies}

The qualitative and quantitative assay of the prepared nano dispersions was carried out using agar well diffusion method. The in-vitro antibacterial assay was performed based on the standards protocol given by National Committee for Clinical Laboratory Standards (NCCLS) protocol. The gram positive and gram-negative strains were used (E.coli, Bacillus and $S$. 
aureus). The samples analysed included the optimised PCs/SOLU nanodispersion (FIII), GSE and standard. The bacterial suspension (inoculum) was prepared by using Mac Farland (108 $\mathrm{CFU} / \mathrm{ml}$ ) media. In the Mueller Hinton agar medium, the prepared inoculum was streaked over the surface and dried. The wells were created using sterile borer and the samples (Gentamycin, GSE, PCs-SOLU) dissolved in water was poured into the wells. Then the plates were kept for incubation at $37^{\circ} \mathrm{C}$ for $24 \mathrm{~h}$. The activity was measured by zone of inhibition developed around the wells. Finally, the images of all the plates were taken after developing zone of inhibition and were used to support the antibacterial activity.

\section{Susceptibility tests by SEM analysis}

The bacterial strains were individually cultured in LB broth and incubated at $37^{\circ} \mathrm{C}$ for $24 \mathrm{~h}$. Then the prepared broth was diluted again with fresh LB broth to reach the optimum density. The glutaraldehyde was dissolved in PBS with $\mathrm{pH} 7.4$ to make $2.5 \% \mathrm{v} / \mathrm{v}$. GSE, gentamycin and GSE-SOLU nanodispersions were taken $(1 \mathrm{mg})$ and kept in LB broth. Bacterial strains were streaked on to the plates and incubated for $24 \mathrm{~h}$ at $37^{\circ} \mathrm{C}$. The glass slides coated with poly lysine were taken and few drops of the samples were added. Then poly lysine coated glass slides were fixed with glutaraldehyde solution and washed with PBS three times. This was followed by storage at $4{ }^{\circ} \mathrm{C}$ and dehydration using ethanol $(70 \%, 96 \%$ and $100 \% \mathrm{v} / \mathrm{v})$. Again the glass slides were dried by critical point dryer (EMS 850) and gold coated for analysing in SEM.

\section{Anti-Susceptibility tests by SEM analysis}

The bacterial strains were individually cultured in LB broth and incubated at $37^{\circ} \mathrm{C}$ for $24 \mathrm{~h}$. Then the prepared broth was diluted again with fresh LB broth to reach the optimum density. The glutaraldehyde was dissolved in PBS with $\mathrm{pH} 7.4$ to make $2.5 \% \mathrm{v} / \mathrm{v}$. GSE, gentamycin and GSE-SOLU nanodispersions were taken $(1 \mathrm{mg})$ and kept in LB broth. Bacterial strains were streaked on to the plates and incubated for $24 \mathrm{~h}$ at $37^{\circ} \mathrm{C}$. The glass slides coated with poly lysine were taken and few drops of the samples were added. Then poly lysine coated glass slides were fixed with glutaraldehyde solution and washed with PBS three times. This was followed by storage at $4{ }^{\circ} \mathrm{C}$ and dehydration using ethanol $(70 \%, 96 \%$ and $100 \% \mathrm{v} / \mathrm{v})$. Again the glass slides were dried by critical point dryer (EMS 850) and gold coated for analysing in SEM.

\section{Anti-inflammatory activity Membrane stabilization test}

Human blood of about $10 \mathrm{ml}$ was collected and kept in the centrifuge tubes and the tubes containing blood were centrifuged for 10 mins. After centrifugation, the samples were washed with saline three times and the volume was measured. Then to the measured volume, saline was added to get a reconstituted suspension of about $10 \% \mathrm{v} / \mathrm{v}$.

\section{Heat-induced haemolytic assay}

The test samples were prepared using $1 \mathrm{ml}$ of reconstituted red blood cells (RBCs) suspension $(10 \%)$ and $1 \mathrm{ml}$ of PCs/SOLU nanodispersion solution. The standards and control were prepared using diclofenac sodium and saline solution $(10 \% \mathrm{v} / \mathrm{v})$. The samples, standard and the control containing centrifuge tubes were incubated for $30 \mathrm{mins}$ at $56^{\circ} \mathrm{C}$. The tubes were cooled and centrifuged for $5 \mathrm{~min}$ at a speed of $2500 \mathrm{rpm}$. All the samples were made in triplicates and the corresponding supernatants was collected. The absorbance was measured at $560 \mathrm{~nm}$. The percentage membrane stabilizing activity was evaluated using the following formula;

Haemolysis Inhibition $(\%)=$ AbsStd - AbsTest AbsStd $\times 100$

\section{Carrageenan and histamine induced rat paw edema}

The method of Winter et al. was utilised with a slight modification[37,38]. Wistar rats was used for this study and were divided into four groups. Each group consist of six animals. For all the animals in all the groups, acute inflammation was induced using phlogistic agents such as carrageenan and histamine $(1 \%$ of $0.1 \mathrm{ml})$ by administration of subcutaneous injection in the hind paw (right side). The paw volume was noted before and after injection to calculate the 
increase or decrease in the inflammation. Then, the diclofenac sodium ointment was administered topically on the hind paw of standard group animals. For the positive and negative control group animals, GSE and the ointment base was applied topically. And for the test group animals, PCs/SOLU nanodispersion ointment was administered on the hind paw topically. After application of ointment, the paw volume was measured continuously every hour for $6 \mathrm{~h}$ using plethysmometer.

\section{Topical semisolid preparation}

The ointment base $(5 \mathrm{~g})$ was prepared by mixing wool fat - $0.250 \mathrm{~g}$, yellow soft paraffin - $4 \mathrm{~g}$, hard paraffin - $0.5 \mathrm{~g}$ and cetosteryl alcohol - $0.250 \mathrm{~g}$ in china dish. Then, the china dish was placed on the water bath which is maintained at a temperature of $50 \mathrm{o}$ till the contents gets liquefied. The quantity required for the preparation of PCs/SOLU nanodispersion ointment is $1 \% \mathrm{w} / \mathrm{w}$ and this was mixed with the melted ointment base and stirred continuously till a homogeneous dispersion was obtained. The same method was followed to prepare GSE ointment.

\section{Excision wound model}

Twenty four adult Wistar rats were divided into four groups of six animals each. The animals were given ketamine intraperitoneal injection as an anaesthetic $(100 \mathrm{mg} / \mathrm{kg})$. The dorsal thoracic area which is $1 \mathrm{~cm}$ away from the vertebral column was cleaned and shaved. After shaving the hair follicles, the area in which the wound was to be created was made circular using stainless steel stencil with methylene blue. The excision wounds was created using toothed forceps, surgical blade and pointed scissors of about $3 \mathrm{~cm}$ in depth. The cotton swab was soaked in saline to clean the wound area and the created wound was left open after attaining haemostasis. The created wound of all the animals was with full thickness which was beneath the adipose tissue. The wound created day was noted and it was considered as day zero and the wound was cleaned daily using methylated spirit before ointment application. Appropriate quantity of ointment base, GSE, Povi/Iod, and PCs/SOLU nanodispersion ointment was applied daily to each individual groups till the complete healing was observed. Area of wound was measured by millimetre scale graph paper and the wound contraction percentage was measured based on the initial wound area. The time taken for complete epithelialisation (i.e., time required for the dead tissue to fall off without any residues) was also measured.

\section{Histopathological analysis of open wound}

The rats were sacrificed on the 12 th day and the skin was excised from the wound area. The excised skin were kept in isotonic saline solution and in deep freezer at $-20{ }^{\circ} \mathrm{C}$ for storage. Using Leica CM1950 cryostat cutter, the excised skin were cut into $5 \mu \mathrm{m}$ thin sections. Eight skin sections of each samples were prepared. Using Leica tissue freezing medium (Germany), the sectioned skin was fixed in the glass slides. Each sample containing eight skin sections were prepared and stained by Leica AutostainerXL using hematoxylin and eosin. After staining procedure, the sectioned samples were visualised using Leica compound microscope (DF2500) which is fitted with camera to capture illustrative images.

\section{Statistical analysis}

The statistical evaluation was performed by using ANOVA and Turkey Krammer Multiple Comparison Test. The $\mathrm{p}<0.05$ value was observed to be statistically significant.

\section{Results and Discussion \\ PCs loading}

PCs (PCs - 1\%) -SOLU were prepared with different theoretical SOLU loading, i.e., $1 \%$ (FI), $3 \%$ (FII), $5 \%$ (FIII) and $7 \%$ (FIV) of polymer. The particle size of the FIII formulation was found to be the smallest particle size $(69.90 \pm 2.12 \mathrm{~nm})$ with PDI of $0.154 \pm 0.023$ (Fig 1 (a)). The polymer SOLU loading of $5 \%$ (FIII) resulted in stable formulation with highest loading efficiency $(95.36 \% \pm 2.06 \%)$ and negative zeta potential $(-82.1 \pm 1.07)$ (Fig 1 (b)). Therefore, the formulation (FIII) was selected for further physicochemical characterization. 

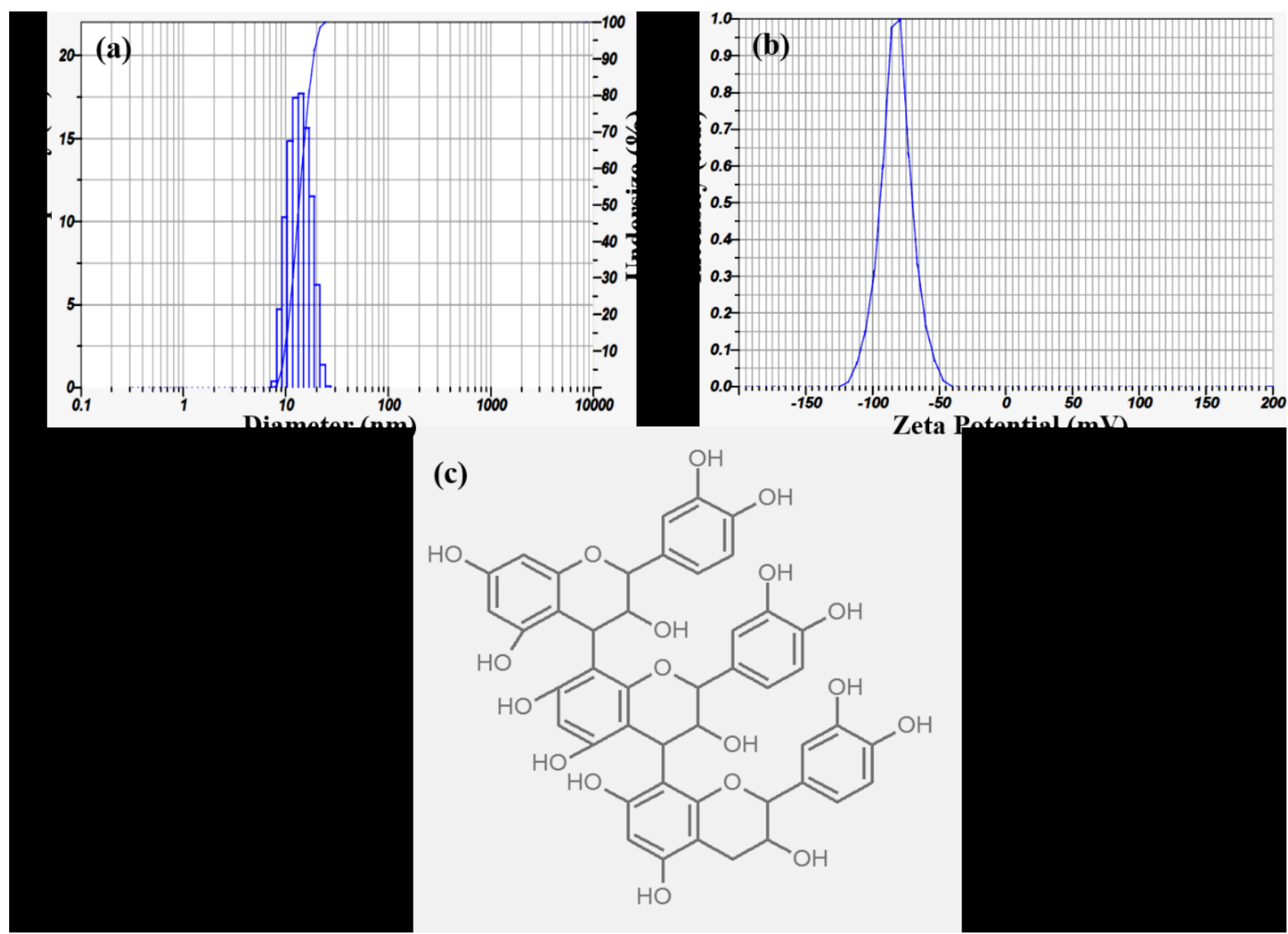

Fig 1 (a) Particle size, (b) Zeta potential of FIII formulation and (c) Chemical structure of proanthocyanidins (PCs).

10

\section{Optical and structural analyses}

The PCs/SOLU nanodispersion formulations were characterized by XRD analysis, UV-Vis analysis, FT-IR analysis, particle size, loading efficiency and TEM analysis (Fig 2). PCs exhibited a peak around 270 to $280 \mathrm{~nm}$ (Fig 2 (a)) which represents the $\pi$ - $\pi *$ transition caused by the presence of double bond (unsaturation). The peak is completely absent in SOLU due to the absence of unsaturation. In addition, the intensity of $\pi-\pi *$ peak of PCs decreases with the addition of SOLU. This is due to the $\pi-\pi$ interactions coming from keto part of SOLU and double bond from the PCs. In the FT-IR spectra, the position of the carbonyl peak in the FIII formulation shifted to $1738 \mathrm{~cm}-1$ and this shifting leads to polar-polar interactions. The FIII formulation, also shows a peak broadening from $3619 \mathrm{~cm}^{-1}$ to $3265 \mathrm{~cm}^{-1}$ which indicates the formation of intermolecular hydrogen bonding between PCs and SOLU (Fig 2 (b)). The diffraction pattern of the SOLU and PCs showed broad peaks at 20 and 22 indicating their amorphous nature. The addition of SOLU to the PCs completely eliminates the broad peak indicating hydrogen bonding and $\pi-\pi$ interactions. The FIII formulation PCs/SOLU dispersion showed no characteristic peak which indicates completely amorphous nature (Fig 2 (c)). The TEM images showed that SOLU (Fig 2 (d)) has a flat thin sheet-like morphology while PCs (Fig 2 (e)) appears as spherical shaped structure. It is clearly evident from the FIII formulation micrograph Fig 2 (f) that the spherically shaped particles of PCs are embedded on the surface of the polymer SOLU. 


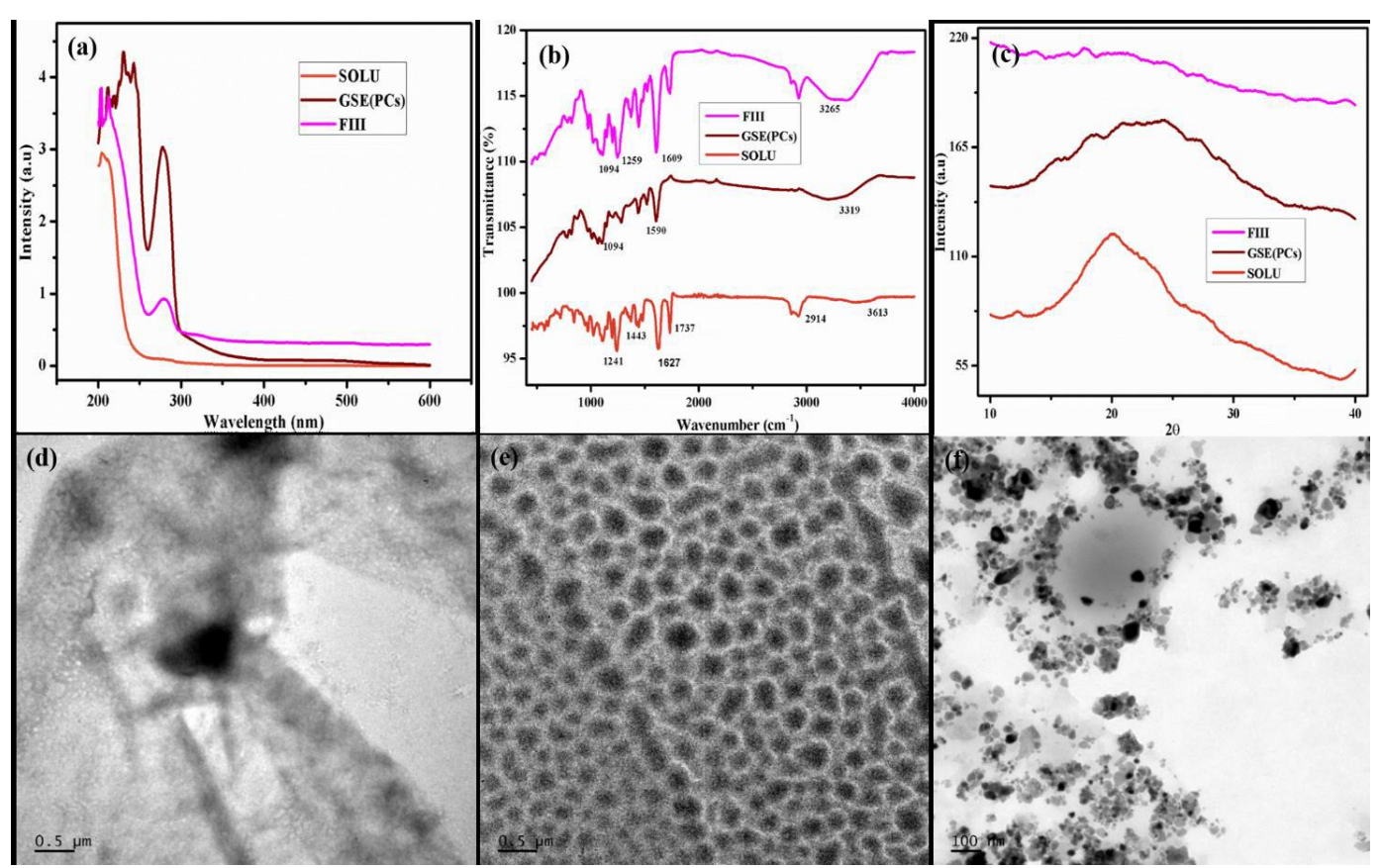

Fig 2 (a) UV-visible spectra, (b) FTIR spectra and (c) XRD pattern of SOLU, PCs and FIII formulation and TEM image of (d) Soluplus (SOLU) $(0.5 \mu \mathrm{m})$, (e) Grape seed extract (PCs) $(0.5 \mu \mathrm{m})$ and (f) PCs/SOLU $(100 \mathrm{~nm})$ used for the study.

\section{In-vitro release}

The pure GSE in SIF showed about $97.33 \%$ release in $2 \mathrm{~h}$ and the PCs revealed a controlled release behaviour from SOLU (Fig 3). They exhibited a lower burst effect with $20.00 \%$ in $2 \mathrm{~h}$ and at $48 \mathrm{~h}$ about $68.05 \%$ was released in controlled manner. The reason for the controlled release profile of PCs was due to the interaction or affinity between the polymer and SOLU. The PCs shows about 45-50 \% of GSE released in $6 \mathrm{~h}$ time period. Similar result had been reported by Lu et al's work for the procyanidins release in tea after $6 \mathrm{~h}$ [39].

\section{Antibacterial study}

The antimicrobial activity of standard, GSE and GSE-SOLU solid dispersion was tested using the agar well diffusion method (Fig 4 A). The zone of inhibition as seen in Fig 4 B showed the range of 3-6 $\mathrm{mm}$ for standard, 5-8.5 $\mathrm{mm}$ for GSE and 9-13 $\mathrm{mm}$ for GSE-SOLU solid dispersion (FIII). The result of the present investigation highlights the greater antibacterial potential of the solid dispersion than for the plain GSE and the standard drug. The main mechanism for the bactericidal activity of PCs/SOLU nanodispersion is shown in Fig 5. When the nanodispersion, FIII (1:5) was placed in the medium, the polymer matrix become swollen. When PCs/SOLU encountered the positively charged ions in the solution, ion exchange took place, which wasnegative organism which is anaerobic, rod shaped bacteria and it contains fimbria, outer cell membrane with lipopolysaccharides, peptidoglycan layer and inner cytoplasm[40]. By means of electrostatic interactions, the PCs which are positively charged gets attracted to the bacterial cell membrane which is negatively charged. These interactions leads to oxidative damage which makes the cell membrane more permeable. When more PCs enter the cells it damages the genetic material DNA by releasing ROS (reactive oxygen species). The next part explains the susceptibility of bacteria by SEM analysis which confirms the proposed mechanism showing antibacterial activity.

\section{Anti-inflammatory activity}

Table 1 shows the anti-inflammatory activity of PCs/SOLU nanodispersion and standard diclofenac sodium. PCs/SOLU nanodispersion displayed $41.83 \%$ activity, which is almost similar to the percentage heat-induced by haemolysis of diclofenac sodium. Therefore, the antiinflammatory activity of PCs/SOLU and diclofenac shows no significant difference. The main mechanism of the anti-inflammatory action is the inhibition of the metabolism of arachidonic 
acid by preventing the action of COX, lipoxygenase, phospholipase and A2. By inhibiting the action of inflammatory enzymes, PCs/SOLU provides a defensive effect against the wounds inflammatory reactions[45]. As a result, the PCs/SOLU was observed to be an effective antiinflammatory

agent.

\section{Membrane stabilizing activity}

Membrane stabilising activity showed that PCs/SOLU inhibit the lysis of erythrocytes. In general, leucocytes migrates to the inflammation site in response to the stimulus which plays a vital role during inflammatory disorders[46,47]. The PCs/SOLU nanodispersion ointment lowers the occurrence of radicals (super oxide) by preventing the leucocytes migration at the inflammation site. The PCs/SOLU nanodispersion ointment and the diclofenac ointment treated groups shows decreased leucocyte counts. In addition, PCs/SOLU treated animals show higher percentage of inhibition of heat induced by haemolysis of erythrocytes when compared to the diclofenac sodium. They displayed the concentration dependent inhibition of haemolytic activity. Furthermore, PCs/SOLU prevents the rupture of the erythrocytes membrane by releasing the hydrolytic enzymes from lysosome to prevent the damage of the tissues. These membrane stabilization results (Table 1) proves that it is a significant assay for antiinflammatory activity.

The PCs/SOLU ointment and diclofenac sodium ointment were administered to control and treatment groups. Both the ointments inhibited the hind paw edema, which was examined at every hour of the experiment. The results show significant reduction in the histamine induced paw edema. In the first hour after the administration of diclofenac sodium ointment, the maximal paw thickness was observed. Whereas in the case of PCs/SOLU ointment application, a statistically significant inhibition of the edema in the histamine induced assay occurred. This becomes more significant after $2 \mathrm{~h}$ and $3 \mathrm{~h}$ (Fig 9 (a)). The edema and inflammation were caused by $5-\mathrm{HT}$ and histamine which is due to the release of prostaglandins into the inflammation site. This occurred due to the leucocytes migration to the inflamed site. PCs/SOLU inhibited the release of inflammatory mediators such as 5-HT and histamine and thereby inhibiting the edema formation during the first hour. In the next $2 \mathrm{~h}$, they prevented the release of histamine and serotonin, potent vasodilator substances that increases the vascular The PCs/SOLU ointment and diclofenac sodium ointment were administered to control and treatment groups. Both the ointments inhibited the hind paw edema, which was examined at every hour of the experiment. The results show significant reduction in the histamine induced paw edema. In the first hour after the administration of diclofenac sodium ointment, the maximal paw thickness was observed. Whereas in the case of PCs/SOLU ointment application, a statistically significant inhibition of the edema in the histamine induced assay occurred. This becomes more significant after $2 \mathrm{~h}$ and $3 \mathrm{~h}$ (Fig 9 (a)). The edema and inflammation were caused by 5-HT and histamine which is due to the release of prostaglandins into the inflammation site. This occurred due to the leucocytes migration to the inflamed site. PCs/SOLU inhibited the release of inflammatory mediators such as 5-HT and histamine and thereby inhibiting the edema formation during the first hour. In the next $2 \mathrm{~h}$, they prevented the release of histamine and serotonin, potent vasodilator substances that increases the vascular permeability (Fig 9 (b)). In the late phase, PCs/SOLU inhibited the synthesis of prostaglandins which is regarded as the most sensitive phase of anti-inflammatory action. 

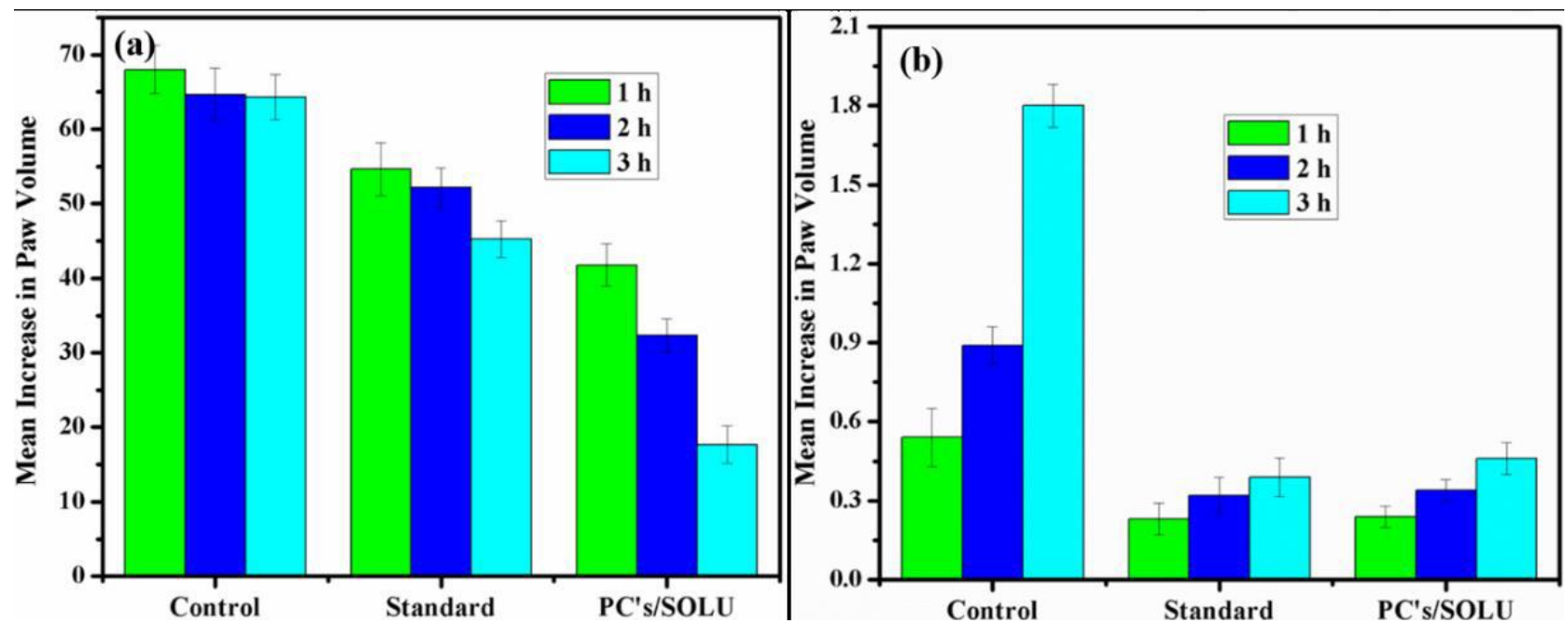

Fig 9 (a) The effects of vehicle (control), diclofenac sodium (standard) and PCs/SOLU in histamine induced rat paw edema and (b) carrageenan induced rat paw edema.

\section{Conclusion}

In this study, PCs/SOLU nanodispersion were successfully prepared and characterized. The prepared PCs/SOLU nanodispersion has polar-polar interactions and indicates the intermolecular hydrogen bonding formation between PCs and SOLU. SEM and TEM analysis confirmed the presence of spherical shaped PCs which were homogeneously distributed in the polymer matrix (SOLU). The antibacterial activity of PCs/SOLU against E.coli, S.aureus and Bacillus proved that the cell membranes became more permeable with disrupted cell structure when treated with nanodispersion. It was evident from the susceptibility tests that the bacterial morphology changed to very small thin structures and blebs were found as aggregates on the cell surface. Carrageenan and histamine induced rat paw edema analyses show there was no inflammatory signs in animals treated with $1 \mathrm{wt} \%$ of PCs/SOLU nanodispersion. Application of PCs/SOLU nanodispersion ointment in wound healing revealed that it improves process of wound healing without scar formation when compared to the control, positive and negative standards. The histopathological assay of PCs/SOLU treated animals showed complete reepithelialisation,

migration of cells, proliferation of cells and fibroblast attachment. Thus, the prepared PCs/SOLU nanodispersion ointment can be used as an effective wound healing component.

\section{Conflicts of interest}

There are no conflicts of interest to declare.

\section{Acknowledgements}

The authors wish to thank the DST-Inspire Fellowship, Department of Science and Technology, New Delhi for their financial support. Authors also express their gratitude to Government of Russian Federation for financially supporting the Project "Agro preparations of the new generation: a strategy of construction and realization" (Agreement No 074-02-2018-328) in accordance with Resolution No 220 "On measures designed to attract leading scientists to the Russian institutions of higher learning". 


\section{References}

[1] Y. Liang, X. Zhao, T. Hu, B. Chen, Z. Yin, P.X. Ma, B. Guo, Adhesive Hemostatic Conducting Injectable Composite Hydrogels with Sustained Drug Release and

Photothermal Antibacterial Activity to Promote Full-Thickness Skin Regeneration

During Wound Healing, Small. 15 (2019) 1- 17. doi:10.1002/smll.201900046.

[2] J. Qu, X. Zhao, Y. Liang, T. Zhang, P.X. Ma, B. Guo, Antibacterial adhesive injectable hydrogels with rapid self-healing, extensibility and compressibility as wound dressing for joints skin wound healing, Biomaterials. (2018).

doi:10.1016/j.biomaterials.2018.08.044.

[3] P.H. Wang, B.S. Huang, H.C. Horng, C.C. Yeh, Y.J. Chen, Wound healing, J. Chinese Med. Assoc. 81 (2018) 94- 101. doi:10.1016/j.jcma.2017.11.002.

[4] J.R. Davidson, Current Concepts in Wound Management and Wound Healing Products, Vet. Clin. North Am. - Small Anim. Pract. 45 (2015) 537- 564.

doi:10.1016/j.cvsm.2015.01.009.

[5] N. Malhotra, S.J. Madison, S.R. Ward, E.R. Mariano, V.J. Loland, B.M. Ilfeld, Continuous Interscalene Nerve Block Following Adhesive Capsulitis Manipulation, Reg. Anesth. Pain Med. 38 (2013) 171- 172. doi:10.1097/AAP.0b013e318283475b.

[6] J. Qu, X. Zhao, P.X. Ma, B. Guo, pH-responsive self-healing injectable hydrogel based on N-carboxyethyl chitosan for hepatocellular carcinoma therapy, Acta Biomater. 58 (2017) 168- 180. doi:10.1016/j.actbio.2017.06.001.

[7] Y. Liang, X. Zhao, P.X. Ma, B. Guo, Y. Du, X. Han, pH-responsive injectable hydrogels with mucosal adhesiveness based on chitosan-grafted-dihydrocaffeic acid and oxidized pullulan for localized drug delivery, J. Colloid Interface Sci. 536 (2019) 224- 234. doi:10.1016/j.jcis.2018.10.056.

[8] X. Zhao, B. Guo, H. Wu, Y. Liang, P.X. Ma, Injectable antibacterial conductive nanocomposite cryogels with rapid shape recovery for noncompressible hemorrhage and wound healing, Nat. Commun. 9 (2018). doi:10.1038/s41467-018-04998-9.

[9] S.M. Ezzat, M.A. Choucry, Z.A. Kandil, Antibacterial, antioxidant, and topical antiinflammatory

activities of Bergia ammannioides: A wound-healing plant, Pharm. Biol.

54 (2016) 215- 224. doi:10.3109/13880209.2015.1028079.

[10] D. Dwivedi, M. Dwivedi, S. Malviya, V. Singh, Evaluation of wound healing, antimicrobial and antioxidant potential of Pongamia pinnata in wistar rats, J. Tradit.

Complement. Med. 7 (2017) 79- 85. doi:10.1016/j.jtcme.2015.12.002.

[11] X. Zhao, H. Wu, B. Guo, R. Dong, Y. Qiu, P.X. Ma, Antibacterial anti-oxidant electroactive injectable hydrogel as self- healing wound dressing with hemostasis and adhesiveness for cutaneous wound healing, Biomaterials. 122 (2017) 34- 47.

doi:10.1016/j.biomaterials.2017.01.011.

[12] J. Qu, X. Zhao, Y. Liang, Y. Xu, P.X. Ma, B. Guo, Degradable conductive injectable hydrogels as novel antibacterial, anti-oxidant wound dressings for wound healing,

Chem. Eng. J. 19 (2019) 548- 560. doi:10.1016/j.cej.2019.01.028.

[13] A.P. Veith, K. Henderson, A. Spencer, A.D. Sligar, A.B. Baker, Therapeutic strategies for enhancing angiogenesis in wound healing, Adv. Drug Deliv. Rev. (2018) 1- 138. doi:10.1016/j.addr.2018.09.010.

[14] S. Saghazadeh, C. Rinoldi, M. Schot, S.S. Kashaf, F. Sharifi, E. Jalilian, K. Nuutila, G. Giatsidis, P. Mostafalu, H. Derakhshandeh, K. Yue, W. Swieszkowski, A. Memic, A. Tamayol, A. Khademhosseini, Drug delivery systems and materials for wound healing applications, Adv. Drug Deliv. Rev. 127 (2018) 138- 166.

doi:10.1016/j.addr.2018.04.008.

[15] R.L. Thangapazham, S. Sharad, R.K. Maheshwari, Phytochemicals in Wound Healing, 
Adv. Wound Care. 5 (2016) 230- 241. doi:10.1089/wound.2013.0505.

[16] N. Kaur, T. Garg, A.K. Goyal, G. Rath, Formulation, optimization and evaluation of curcumin- $\beta$-cyclodextrin-loaded sponge for effective drug delivery in thermal burns chemotherapy, Drug Deliv. 23 (2016) 2245 - 2254. doi:10.3109/10717544.2014.963900.

[17] P.J. Patil, S.P. Chaudhari, S. V. Ghodekar, Evaluation of wound healing activity of silver sulfadiazine emulgel (1\%) in the rat burn wound model and its skin irritation study, Indian Drugs. 49 (2012) 40- 43.

[18] C.O. Esimone, C.S. Nworu, C.L. Jackson, Cutaneous wound healing activity of a herbal ointment containing the leaf extract of Jatropha Curcas L. (Euphorbiaceae), Int. J. Appl.

Res. Nat. Prod. 1 (2008) 1 - 4.

[19] G. Moura-Letts, L.F. Villegas, A. Marçalo, A.J. Vaisberg, G.B. Hammond, In vivo wound-healing activity of oleanolic acid derived from the acid hydrolysis of Anredera diffusa, J. Nat. Prod. 69 (2006) 978- 979. doi:10.1021/np0601152.

[20] M. Heidari, R. Bahramsoltani, A.H. Abdolghaffari, R. Rahimi, M. Esfandyari, M. Baeeri, G. Hassanzadeh, M. Abdollahi, M.H. Farzaei, Efficacy of topical application of standardized extract of Tragopogon graminifolius in the healing process of experimental burn wounds, J. Tradit. Complement. Med. (2018) 1 - 6 .

doi:10.1016/j.jtcme.2018.02.002.

[21] N.R.L. Raja, K. Sundar, Promotion of wound healing by leaf extracts of sesbania aegyptiaca in albino rats, Res. J. Med. Plant. 9 (2015) 116- 126.

doi:10.3923/rjmp.2015.116.126.

[22] S. Mendes Hoepers, H.G.M. Tolentino De Souza, N.L. Meira Quintão, J. Roberto Santin, V. Cechinel Filho, R.M.L. Silva, A. Garcia Couto, K.A.B. Simão Da Silva, Topical anti-inflammatory activity of semisolid containing standardized Aleurites moluccana L. Willd (Euphorbiaceae) leaves extract, J. Ethnopharmacol. 173 (2015) 251 - 255. doi:10.1016/j.jep.2015.07.024.

[23] N. Kittana, H. Abu-Rass, R. Sabra, L. Manasra, H. Hanany, N. Jaradat, F. Hussein, A.N. Zaid, Topical aqueous extract of Ephedra alata can improve wound healing in an animal model, Chinese J. Traumatol. - English Ed. 20 (2017) 108- 113.

doi:10.1016/j.cjtee.2016.10.004.

[24] A. Kundu, A. Ghosh, N.K. Singh, G.K. Singh, A. Seth, S.K. Maurya, S. Hemalatha, D. Laloo, Wound healing activity of the ethanol root extract and polyphenolic rich fraction from Potentilla fulgens, Pharm. Biol. 54 (2016) 2383- 2393. doi:10.3109/13880209.2016.1157192.

[25] S. Rajan, Wound healing activity of an herbal ointment containing the leaf extract of Ziziphus Mauritiana Lam., African J. Pharm. Pharmacol. 7 (2013) 98- 103.

doi:10.5897/AJPP12.795.

[26] V.N. Umeh, E.E. Ilodigwe, D.L. Ajaghaku, E.O. Erhirhie, G.E. Moke, P.A. Akah, Wound-healing Activity of the Aqueous Leaf Extract and Fractions of Ficus exasperata (Moraceae) and its Safety Evaluation on Albino Rats, J. Tradit. Complement. Med. 4 (2014) 246- 252. doi:10.4103/2225-4110.139105.

[27] K. Das, Wound healing potential of aqueous crude extract of stevia rebaudiana in mice, Brazilian J. Pharmacogn. 23 (2013) 351 - 357. doi:10.1590/S0102$695 X 2013005000011$.

[28] S. Khanna, M. Venojarvi, S. Roy, N. Sharma, P. Trikha, D. Bagchi, M. Bagchi, C.K. Sen, Dermal wound healing properties of redox-active grape seed proanthocyanidins, Free Radic. Biol. Med. 33 (2002) 1089- 1096. doi:10.1016/S0891-5849(02)00999-1. [29] H. Nejati, M.R. Farahpour, Effect of topical red grape seed hydroethanol extract on burn wound healing in rats, Int. J. ChemTech Res. 6 (2014) 2340- 2346.

[30] J.P. Heggers, J. Cottingham, J. Gusman, L. Reagor, L. McCoy, E. Carino, R. Cox, J.-G. 
Zhao, L. Reagor, The effectiveness of processed grapefruit-seed extract as an antibacterial agent: I. An In Vitro Agar Assay, J. Altern. Complement. Med. 8 (2002) 333 - 40. doi:10.1089/10755530260128023.

[31] S. Soorgi, A.M. Izadpanah, G.R. Sharifzadeh, M. Torshizi, Z. Khazaye, M. Hasanpour, The Effects of Grape Seed Extract Ointment on Approximation of Cesarean Section Wound, Mod. Care J. 13 (2016) 1- 6. doi:10.17795/modernc.8849.Research. [32] A.A. Hemmati, M. Foroozan, G. Houshmand, Z.B. Moosavi, M. Bahadoram, N.S. Maram, The Topical Effect of Grape Seed Extract 2\% Cream on Surgery Wound Healing, Glob. J. Health Sci. 7 (2014) 52- 58. doi:10.5539/gjhs.v7n3p52.

[33] S.M. Attia, S.A. Bakheet, N.M. Al-Rasheed, Proanthocyanidins Produce Significant Attenuation of Doxorubicin-Induced Mutagenicity via Suppression of Oxidative Stress, Oxid. Med. Cell. Longev. 3 (2010) 404- 413. doi:10.4161/oxim.3.6.14418.

[34] S. Khanna, S. Roy, D. Bagchi, M. Bagchi, C.K. Sen, Upregulation of oxidant-induced VEGF expression in cultured keratinocytes by a grape seed proanthocyanidin extract, Free Radic. Biol. Med. 31 (2001) 38- 42. doi:10.1016/S0891-5849(01)00544-5.

[35] A.A. Hemmati, N. Aghel, I. Rashidi, A. Gholampur-Aghdami, Topical grape (Vitis vinifera) seed extract promotes repair of full thickness wound in rabbit, Int. Wound J. 8 (2011) 514- 520. doi:10.1111/j.1742-481X.2011.00833.x.

[36] Z. Wang, X. Ju, R. He, J. Yuan, R.E. Aluko, Effect of high pressure treatment on rapeseed protein microparticle properties and gastrointestinal release behavior of the encapsulated peptides, Food Res. Int. 77 (2015) 549- 555.

[37] C.A. Winter, Carrageenin-Induced Edema in Hind Paw, Antiinflammatory Assay. (1984) 544- 547.

[38] A.U. Tatiya, A.K. Saluja, Further studies on membrane stabilizing, anti-inflammatory and FCA induced arthritic activity of various fractions of bark of machilus macrantha in rats, Brazilian J. Pharmacogn. 21 (2011) 1052- 1064. doi:10.1590/S0102695X2011005000152.

[39] Q. Lu, D.-C. Li, J.-G. Jiang, Preparation of a tea polyphenol nanoliposome system and its physicochemical properties, J. Agric. Food Chem. 59 (2011) 13004- 13011.

[40] R.D. Turner, A.F. Hurd, A. Cadby, J.K. Hobbs, S.J. Foster, Cell wall elongation mode in Gram-negative bacteria is determined by peptidoglycan architecture, Nat. Commun. 4 (2013) 1496- 1498. doi:10.1038/ncomms2503.

[41] C. Tang, B. Xie, Z. Sun, Antibacterial activity and mechanism of B-type oligomeric procyanidins from lotus seedpod on enterotoxigenic Escherichia coli, J. Funct. Foods. 38 (2017) 454- 463. doi:10.1016/j.jff.2017.09.046.

[42] S.K.H. and G.J.P. N. Savitri Kumar, B. M. Ratnayake Bandara, Oligomeric proanthocyanidin fractions from fresh tea leaves and Staphylococcus aureus, J.Natn.Sci.Foundation. 42 (2014) 241 - 246. doi:http://dx.doi.org/10.4038/jnsfsr.v42i3.7397.

[43] V.S. Chedea, C. Braicu, F. Chirilə, H. J. 0. Ogola, R. Ş. Pelmuş, L. G. Cəlin, C. Socaciu, Antioxidant/Prooxidant and Antibacterial/Probacterial Effects of a Grape Seed Extract in Complex with Lipoxygenase, Biomed Res. Int. 2014 (2014) 1- 9. doi:10.1155/2014/313684.

[44] X. Li, C. He, L. Song, T. Li, S. Cui, L. Zhang, Y. Jia, Antimicrobial activity and mechanism of Larch bark procyanidins against Staphylococcus aureus, Acta Biochim. Biophys. Sin. (Shanghai). 49 (2017) 1058- 1066. doi:10.1093/abbs/gmx112.

[45] T.X. LI Wen-Guang, Zhang Xiao-Yu, WU Yong-Jie, Anti-inflammatoryeffect and mechanism of proanthocyanidins from grape seeds, Acta Pharmacol. Sin. 22 (2001) 1117- 1120.

[46] Reshma, K.P. Arun, P. Brindha, In vitro anti-inflammatory, Antioxidant and 
nephroprotective studies on leaves of Aegle marmelos and Ocimum sanctum, Asian J. Pharm. Clin. Res. 7 (2014) 121- 129. doi:10.1002/dvdy.22691.

[47] L. Kambizi, M.T. Bakare-Odunola, A.T. Oladiji, A.T. Kola-Mustapha, T.O. Amusa, O. Atolani, N.S. Njinga, A.L. Quadri, Proteinease inhibition, membrane stabilization, antioxidant and phytochemical evaluations of leaves, seeds and calyces of four selected edible medicinal plants, Cogent Chem. 3 (2017) 1- 15. doi:10.1080/23312009.2017.1314064.

[48] D. Ferreira, D. Slade, Oligomeric proanthocyanidins : naturally occurring Oheterocycles, Nat. Prod. Rep. 19 (2002) 517- 541. doi:10.1039/b008741f.

[49] D.K. Das Debasis Bagchi, Manashi Bagchi, Sidney J. Stohs, C.A.K. Sidhartha D. Ray, S.S.J. D, H.G.P. E, Free radicals and grape seed proanthocyanidin extract : Importance in human health and disease prevention Free radicals and grape seed proanthocyanidin extract : importance in human health and disease prevention, Toxicology. 148 (2000) 187-197. doi:10.1016/S0300-483X(00)00210-9. 